\title{
Genetic Polymorphism between Tobacco Cultivar-groups Revealed by Amplified Fragment Length Polymorphism Analysis
}

\author{
Jessada Denduangboripant (Corresponding author) \\ Department of Biology, Faculty of Science, Chulalongkorn University \\ Phayathai road, Bangkok, 10330, Thailand \\ Tel: 66-2218-5378Ｅ-mail: jessada.d@chula.ac.th
}

Tianrat Piteekan

Biotechnology Program, Faculty of Science, Chulalongkorn University

Phayathai road, Bangkok, 10330, Thailand

Tel: 66-2218-5368Ｅ-mail: teanrat@hotmail.com

Matchima Nantharat

Biotechnology Program, Faculty of Science, Chulalongkorn University

Phayathai road, Bangkok, 10330, Thailand

Tel: 66-2218-5368 E-mail: my_matchima@hotmail.com

This research study was supported by Thailand Tobacco Monopoly, Ministry of Finance.

\begin{abstract}
Tobacco (Nicotina tabacum) has been introduced to Thailand for hundreds of years. All tobaccos cultivated in the country are legally separated to local (or early-imported) and imported cultivar groups. However, no method could precisely differentiate the two groups, especially from cured leaf samples. Amplified fragment length polymorphism (AFLP) analysis was introduced to estimate genetic polymorphism of 19 tobacco cultivars grown in Thailand. Thirty-two selective primer-combinations were screened on the genomic DNA extracted from cured leaves. Three primer pairs were selected and resulted in 139 scorable AFLP fragments, of which 103 (74.1\%) were polymorphic. Genetic relationship analysis revealed clustering patterns of tobacco samples generally following the cultivar groups. Almost all local cultivars were found closely related to Burley and Turkish types of the imported group, but significantly separated from Virginia type. Our finding therefore should be an important knowledge for further research on cultivar identification and genetic improvement of tobaccos.
\end{abstract}

Keywords: Amplified fragment length polymorphism, Genetic polymorphism, Molecular marker, Thailand, Tobacco.

\section{Introduction}

\subsection{Local and imported tobacco-groups}

Tobacco is a perennial plant in the genus Nicotiana of the family Solanaceae. The genus Nicotiana contains about 64 different species of herbs and shrubs (Goodspeed, 1954). Almost all of the tobaccos cultivated worldwide belong to the species $N$. tabacum $\mathrm{L}$ and its leaves are commercially processed as an ingredient of cigarettes and cigars. Nicotiana tabacum is a natural amphidiploid $(2 \mathrm{n}=48)$ derived from hybridisation between $N$. sylvestris and N. tomentosiforimis wild progenitor species (Gerstel, 1960 and Gerstel, 1963). Tobacco originated in the tropical Americas and now it can be found cultivated in almost all subtropical and temperate regions of the world from about $60^{\circ}$ North to $45^{\circ}$ South (Akehurst, 1981). Currently, tobacco has been one of major economic forces in almost one hundred countries around the world (Ren \& Timko, 2001). It is also known to be the most widely-grown non-food crop in the world.

Tobacco has become one of the most important crops of Thailand, commercially grown in various regions especially in the North, Northeast and Central. It is believed to be introduced to the country prior to the $16^{\text {th }}$ 
century in the era of King Narai the Great. Monsieur De La Loubare, a royal envoy from King Louis the $14^{\text {th }}$ of the French monarchy, described in his famous annals that he saw both Thai men and women smoking rolled tobaccos. Tobaccos were probably first imported to Thailand from Manila and China in the Sukhothai era (around $12^{\text {th }}$ century) and were then widely cultivated in the northern and northeastern regions. More recent record of tobaccos imported to Thailand started when British American Tobacco company (B.A.T.) introduced Virginia-type cultivars (or flue-cured tobacco) in 1935. Thereafter, the Royal Thai government took over all operations of B.A.T. and other tobacco companies and founded Thailand Tobacco Monopoly in 1939. Since then the company has imported tobacco seeds from western countries for research and development of tobacco cultivation. Burley (or lightly air-cured tobacco) and Turkish (or sun-cured tobacco) types were first imported during 1958-1959 to be a filler material in their cigarette production.

Following Thailand's tobacco law, all tobaccos grown in the country are classified into either "local" or "imported" cultivar groups. The two groups have great differences in crop-growing permission and excise-collecting regulations. For instance, an excise charge for any product from local tobacco cultivars is almost none while the charge for imported cultivars is a hundred times higher. However, there is no precise method, either physical or chemical, to confidently suggest whether the tobacco leaves are of local or imported cultivar group. A common practice at the moment is to examine the leaf size and shape, leaf texture, smoking smell and taste. Nevertheless, all of these conventional methods could be totally misleading depending on cultivating and leaf-curing conditions of the tobaccos. Moreover, a very concise, but legal definition of the local cultivar as "early-imported tobaccos to Thailand long time ago" is also confusing and not testable.

\subsection{AFLP molecular marker}

Such problem may be solved by introducing molecular marker techniques to determine genetic differences between tobacco cultivar groups. During the last two decades, PCR-based molecular markers such as random amplified polymorphic DNA (RAPD), inter-simple sequence repeat (ISSR) and amplified fragment length polymorphism (AFLP) have played an important role in genetic polymorphism investigation of tobaccos. RAPD marker was first introduced to identify tobacco hybrids (de Filippis et al., 1996) and later used to analyse genetic diversity between cultivars (del Piano et al., 2000; He et al., 2001; Rossi et al., 2001; Arslan \& Okumus, 2005; Sarala \& Rao, 2008 and Zhang et al., 2008). ISSR marker was also used to study tobacco genetic diversity (Yang et al., 2005) and in genetic fingerprinting of Nicotiana spp. (del Piano et al., 2004). Likewise, AFLP has been successfully used in genetic analysis of tobacco cultivars (Ren \& Timko, 2001; Rossi et al., 2001; Zhang et al., 2006, Siva Raju et al., 2008 and Zhang et al., 2008) and it is better than RAPD or ISSR for a narrow-genetic basis of cultivated crops because of its higher number of polymorphic loci per primer (Bogani et al., 1997). Furthermore, AFLP appears to be an appropriate method for processed tobacco leaves (Rossi et al., 2001).

Previously, we introduced ISSR method to preliminarily examine whether the genetic variation of tobacco cultivars grown in Thailand is high enough to distinguish the local cultivar group (Denduangboripant, 2010). Several ISSR markers were developed and revealed that some local samples were distantly separated from other cultivars, suggesting their early history of introduction into Thailand. Nevertheless, we found that the ISSR technique was not efficiently used with cured tobacco leaves. Therefore, AFLP technique was introduced in this study and we successfully developed AFLP markers suitable to determine genetic differences between local and imported tobacco cultivar groups.

\section{Material and Methods}

\subsection{Tobacco leaf samples}

Cured leaf specimens of 19 tobacco cultivars were collected to analyse in this study with the help of Thailand Tobacco Monopoly, Ministry of Finance (Table 1). Eleven local and five imported cultivars were sampled in crop fields from 12 different provinces of all regions in Thailand. Additionally, three more imported cultivars were obtained from Maejo Tobacco Experiment Station in Chiang Mai province. The leaf samples were dried and kept separately in plastic bags full with silica gel until DNA preparation.

\subsection{DNA extraction and AFLP-PCR amplification}

The tobacco leaves were ground to fine powder with liquid nitrogen and total genomic DNA was extracted using Plant Genomic DNA Mini kit (Geneaid, Taiwan) following an instruction of the manufacturer. AFLP amplification was performed based on the protocol of Vos et al. (1995) with some modification. Approximately $250 \mathrm{ng}$ of the extracted DNA was digested completely at $37^{\circ} \mathrm{C}$ with 6 units of EcoRI restriction enzyme in a total volume of $25 \mu \mathrm{l}$, and subsequently digested with 3 units of Tru9I (an isoschizomer of MseI) enzyme. Oligonucleotide adapters of EcoRI (5'-CTC GTA TGC GTA CC-3') and MseI (5'-AAT TGG TAC GCA GTC 
TAC-3') were ligated to the DNA fragments in a total volume of $20 \mu \mathrm{l}$ with 1 unit of T4 DNA ligase at $4^{\circ} \mathrm{C}$ for 16 hours. Pre-selective amplification step was carried out using adapter-specific primers with a single selective nucleotide: primer $\mathrm{E}_{\mathrm{A}}$ (5'-GAC TGA GTA CCA ATT CA-3'; for EcoRI adapter) and $\mathrm{M}_{\mathrm{C}}$ (5'-GAT GAG TCC TGA GTA AC-3'; for MseI).The following PCR condition was employed: 20 cycles of $30 \mathrm{~s}$ at $94^{\circ} \mathrm{C}, 60 \mathrm{~s}$ at $56^{\circ} \mathrm{C}$ and $60 \mathrm{~s}$ at $72^{\circ} \mathrm{C}$. The pre-amplified product was diluted in the ratio of 1:20 and used as a template for selective amplification using adapter-specific primers with three selective nucleotides. Thirty-two primer combinations were screened for the ones that could produce high numbers of clearly scorable polymorphic bands. The cycling parameters of this step were: first cycle of $30 \mathrm{~s}$ at $94^{\circ} \mathrm{C}, 30 \mathrm{~s}$ at $67^{\circ} \mathrm{C}$ and $60 \mathrm{~s}$ at $72^{\circ} \mathrm{C}$, and lowering the annealing temperature by $0.7^{\circ} \mathrm{C}$ per cycle for another 11 cycles, followed by 23 cycles of $94^{\circ} \mathrm{C}$ for $30 \mathrm{~s}, 56^{\circ} \mathrm{C}$ for $30 \mathrm{~s}$ and $72^{\circ} \mathrm{C}$ for $60 \mathrm{~s}$. The AFLP amplified products were separated by electrophoresis on $6 \%$ denaturing polyacrylamide gel containing $7 \mathrm{M}$ urea and visualised by silver staining. Sizes of the fragments were estimated using 50- and 100-basepair (bp) DNA ladder markers.

\subsection{Genetic relationship analysis}

The AFLP bands were treated as dominant markers and only bright, clearly-resolved AFLP fragments were scored for presence (1) or absence (0) of the bands. Nei and Li (1979)'s coefficient analysis was used to calculate pairwise band-similarity values of the samples using program PAUP* 4.0b10 (Swofford, 2002). A cluster analysis was performed to construct a tree diagram using unweighted pair group method with arithmetic mean (UPGMA) (Sneath \& Sokal, 1973) method. Reliability of the clusters on the tree was estimated by bootstrap analysis with 1,000 replications to show the degree of confidence of each branch. Only bootstrap values over $50 \%$ were considered significant. The clustering patterns were compared with other information of the tobacco cultivars.

\section{Results and discussion}

\subsection{AFLP amplification results}

From our AFLP study on the cured leaf samples of 19 tobacco cultivars, three of 32 screened primer-combinations ( $\mathrm{E}_{\mathrm{AAG}} / \mathrm{M}_{\mathrm{CAA}}, \mathrm{E}_{\mathrm{AAG}} / \mathrm{M}_{\mathrm{CGC}}$ and $\mathrm{E}_{\mathrm{ACT}} / \mathrm{M}_{\mathrm{CAG}}$ ) were found suitable for the selective amplification step. Sizes of the AFLP fragments amplified with the three primer-pairs ranged from 100 to 750 basepairs (see an example in Figure 1). The AFLP fingerprints gave a total number of 139 scorable bands with an average number of 46 fragments per primer pair (Table 2). There were totally 103 polymorphic bands and the average polymorphism degree was 34 polymorphic loci per primer pair. The highest degree of AFLP polymorphism (83.1\%) was generated by $\mathrm{E}_{\mathrm{ACT}} / \mathrm{M}_{\mathrm{CAG}}$ primer combination. Moreover, the $\mathrm{E}_{\mathrm{ACT}} / \mathrm{M}_{\mathrm{CAG}}$ primer pair could generate one major band (approximately $150 \mathrm{bp}$ ) specific to Virginia tobacco type (see lanes 1-3 in Figure 1). This 150-bp Virginia-specific band was similar in length to that of Lao-dong cultivar (lane 22), but the latter was slightly smaller.

The average number of the amplified AFLP fragments in this research (46 per primer pair) was rather low compared to those of previous studies. For example, Ren and Timko (2001) successfully detected 92 amplified bands per primer in their AFLP analysis of 46 cultivated tobacco accessions. Zhang et al. (2006) produced 82 AFLP-PCR fragments per primer from 51 Virginia-type tobacco cultivars. Moreover, an AFLP study on 54 tobacco cultivars in India could amplify AFLP products in the ratio of 84 fragments per primer (Siva Raju et al., 2008). In fact, these three previous studies used fresh tobacco leaves for their experiments. Thus, our smaller number of the AFLP fragments may have resulted from low quality of the genomic DNA extracted from cured leaf materials.

Fortunately, a 150-bp Virginia-type specific band was found from the $\mathrm{E}_{\mathrm{ACT}} / \mathrm{M}_{\mathrm{CAG}}$ AFLP profile (Figure 1). More number of tobacco cultivars should be examined in the future to confirm specificity and efficiency of this Virginia-specific marker. Even though Siva Raju et al. (2008) revealed as many as 34 species-specific markers for N. tabacum species, they could not find any specific band for tobacco cultivars. Ren and Timko (2001) also suggested that an AFLP technique may not be effective enough to analyse genetic polymorphism at the subspecies level in the genus Nicotiana. Therefore, we would take this great opportunity to further develop a sequence charecterised amplified region (SCAR) marker for Virginia cultivars. Such SCAR marker could be legally implemented as a quick, simple tool to examine any suspicious tobacco product whether it comes from a local or imported (only Virginia-type) cultivar group.

\subsection{Genetic relationship from UPGMA analysis}

From the UPGMA genetic tree (Figure 2), almost all 11 local tobacco cultivars were grouped together, except Hangkai which was positioned distantly from the others. Five local cultivars sampled from the central region of 
Thailand (Bai-lai, Kan, Kariang, Bai-tang and Laodong) were clustered on the tree. Within this group, a subgrouping of Bai-lai, Kan and Kariang cultivars was found with $54 \%$ bootstrap supporting value whereas a pair of Bai-tang and Lao-dong was strongly supported with $93 \%$ bootstrap. Another major cluster of the local cultivars composed of White gold, K326 Phuen-mueang, E-dum and Ya-glai. The first three cultivars were also significantly grouped together with $67 \%$ bootstrap support. In the case of the imported cultivar group, Samsun and Xanthiyaka (Turkish type) tobacco cultivars were paired together, but Samsun was also mixed with TN97 (Burley) with very high 95\% bootstrap value. The other two Burley cultivars, TN86 and TN90, were paired together and placed near to Hangkai local cultivar. Interestingly, K326, PV09 and PVH03 (Virginia) cultivars were robustly grouped and distinctively separated from the other imported cultivars with $100 \%$ supporting value.

Our UPGMA genetic tree suggested that most of the local cultivars were closely related with each other and also with the imported cultivars, particularly Burley and Turkish types. The subgroupings of some tobacco cultivars on the UPGMA tree would have followed the cultivar types and the cultivating areas. Our hypothesis agrees well with some previous AFLP studies on tobacco genetic polymorphism. For instance, Ren and Timko (2001) reported that 46 tobacco cultivars representing 18 different countries around the world could be divided primarily based on geographic origins and manufacturing quality traits. They also suggested that the genetic variation among cultivated tobacco lines was limited as evidenced by the high degree of genetic similarity. Moreover, Zhang et al. (2006) found that 51 Virginia cultivars with desirable agronomic characteristics (such as high leaf yield, low nicotine content and resistance to various diseases) also formed groupings based on their geographic origin. Likewise, 16 Indian tobacco cultivars used in cigarette manufactures, especially the bidi type, were clustered together in the AFLP study of Siva Raju et al. (2008).

\subsection{Genetic polymorphism between tobacco cultivar-groups}

Regarding to the geographic influence on genetic relationships among tobacco cultivars, Bai-lai, Kariang, Kan, Bai-tang and Lao-dong would have grouped together due to their common origin of cultivation in the central region of Thailand. Another cluster of White gold, K326 Phuen-mueang, E-dum, Phu and Ya-glai cultivars may suggest a similar scenario, but originated in the Northeast. Nevertheless, both subgroups of local cultivars were placed closely to Burley and Turkish types of the imported cultivars, suggesting their common origin as well. This finding agrees well with the results of our previous ISSR analysis (Denduangboripant et al., 2010) in which most of the local cultivars grown in Thailand were closely related to Burley and Turkish cultivars. Both tobacco types were first imported around 50 years ago and they have been distributed to farmers almost every year. These imported tobacco cultivars have been grown in rural areas for many decades and eventually obtained new Thai names. In this case, such tobacco cultivars should be more appropriately pronounced as "recently-imported cultivars". On the other hand, we could call Hangkai cultivar as "a true local cultivar" because it was positioned distantly from the other tobaccos, suggesting a very low degree of genetic similarity.

Similarly to Hangkai local cultivar, the three Virginia-type cultivars - K326, PV09 and PVH03 - were distinctively separated from other imported and local cultivars. This finding implies that the genetic differentiation between Virginia cultivars and the other tobaccos is probably much higher than we previous thought. Our suggestion agrees well with the AFLP results of Siva Raju et al. (2008) in which the cultivated Virginia cultivars were clustered separately from Burley cultivars. The RAPD analysis of Sarala and Rao (2008) also revealed similar results by clearly distinguishing two Burley cultivars from eight Virginia cultivars.

\section{Conclusion}

In this study, three selective AFLP primer-pairs were selected to study genetic differentiation between cultivar groups of 19 tobacco cultivars. The genomic DNA extracted from cured leaf samples gave clear AFLP-PCR profiles with a high number of the scorable AFLP bands. The UPGMA tree revealed several subgroupings of the cultivars which tended to follow their cultivar-types and growing regions. Most of the local cultivars in Thailand were grouped together but also closely related to Burley and Turkish types of the imported cultivars. Virginia-type cultivars were distinguished from other imported cultivars and the Virginia-specific amplified marker was also found. Therefore, our AFLP technique could be used to differentiate the local tobacco cultivar-group from the imported group, especially to the Virginia-type cultivars. The findings from this research should be an important source of knowledge for tobacco genetic studies in the future, such as genotyping of tobacco cultivars, germplasm improvement, and parental selection for breeding purposes.

\section{Acknowledgements}

The first author was sponsored by research grants (no. MRG-4680001) of Thailand Research Fund (TRF). The second and third authors were also supported by TRF graduate student grants (no. MRG-OSMEP505S009 and no. MRG-OSMEP505S010, respectively). We would like to thank Chiwin Kunalai, Wilasinee Suwanprasart and 
other officers of Thailand Tobacco Monopoly for their great help in tobacco leaf sampling and research collaboration.

\section{References}

Akehurst, B. C. (1981). Tobacco. New York: Longman.

Arslan, B., \& Okumus, A. (2006). Genetic and geographic polymorphism of cultivated tobacco (Nicotiana tabacum) in Turkey. Russian Journal of Genetics, 42, 667- 671.

Bogani, P., Liò, P., Intrieri, M. C., \& Buiatti, M. (1997). A physiological and molecular analysis of the genus Nicotiana. Molecular Phylogenetics and Evolution, 7, 62-70.

de Filippis, L., Hoffman, E., \& Hampp, R. (1996). Identification of somatic hybrids of tobacco generated by electrofusion and culture of protoplasts using RAPD-PCR. Plant Science, 121, 39-46.

del Piano, L., Abet, M., Sorrentino, C., Acanfora, F., Cozzolino, E., \& Di Muro, A. (2000). Genetic variability in Nicotiana tabacum and Nicotiana species as revealed by RAPD procedure. Beiträge zur tabakforshung International Contribution to Tobacco Research, 19, 1-15.

Denduangboripant, J., Setaphan, S., Suwanprasart, W., \& Panha, S. (2010). Determination of local tobacco cultivars using ISSR molecular marker. Chiang Mai Journal of Science, 37(2), 001-011.

Gerstel, D. U. (1960). Segregation in new allopolyploids of Nicotiana. I. Comparison of 6x (N. tabacum x tomentosiformis) and 6x (N. tabacum $\mathrm{x}$ otophora). Genetics, 45, 1723-1734.

Gerstel, D. U. (1963). Segregation in new allopolyploids of Nicotiana. II. Discordant ratios from individual loci in 6x (N. tabacum x N. sylvestris). Genetics, 48, 677-689.

Goodspeed, T. H. (1954). The Genus Nicotiana. Massachusetts: Chronica Botanica.

He, C. S., He, X. J., Ge, S., Li, T. F., Xu, L. Y., Xu, M. L., \& Xu, J. M. (2001). Analysis of germplasm of flue-cured tobacco by RAPD. Acta Botanica Sinica, 43, 610-614.

Nei, M., \& Li, W. H. (1979). Mathematical model for studying genetic variation in term of restriction endonucleases. Proceedings of the National Academy of Sciences of the USA, 76, 5269-5273.

Ren, N., \& Timko, M. P. (2001). AFLP analysis of genetic polymorphism and evolutionary relationships among cultivated and wild Nicotiana species. Genome, 44, 559-571.

Rossi, L., Bindler, G., Pijnenburgh, H., Isaac, P. G., Giraud-Henry, I., Mahe, M., Orvain, C., \& Gadani, F. (2001). Potential of molecular marker analysis for variety identification in processed tobacco. Plant Varieties and Seeds, 14, 89-101.

Sarala, K., \& Rao, R. V. S. (2008). Genetic diversity in Indian FCV and Burley tobacco cultivars. Journal of Genetics, 87, 159-163.

Siva Raju,. K., Madhav, M. S., Sharma, R. K., Murthy, T. G. K., \& Mohapatra, T. (2008). Genetic polymorphism of Indian tobacco types as revealed by amplified fragment length polymorphism. Current Science, 94, 633-639.

Sneath, P. H. A., \& Sokal. R. R. (1973). Numerical Taxonomy: the Principle and Practice of Numerical Classification. San Francisco: Freeman.

Swofford, D. L. (2002). PAUP*: Phylogenetic Analysis Using Parsimony (*and Other Methods) version 4.0b10. Massachusetts: Sinauer Associates.

Vos, P., Hogers, R., Bleeker, M., Reijans, M., van de Lee, T., Hornes, M., Frijters, A., Pot, J., Peleman, J., Kuiper, M., \& Zabeau, M. (1995). AFLP: a new technique for DNA fringerprinting. Nucleic Acids Research, 23, 4407- 4414.

Yang, B. C., Xiao, B. G., Chen, X J., \& Shi, C.H. (2005). Genetic diversity of flue-cured tobacco varieties based on ISSR markers. Hereditas (Beijing), 27, 753-758.

Zhang, H. Y., Liu, X. Z., He, C. S., \& Yang, Y. M. (2008). Genetic diversity among flue-cured tobacco based on RAPD and AFLP markers. Brazilian Archives of Biology and Technology, 51, 1097-1101.

Zhang, H. Y., Liu, X. Z., Li, T. S., \& Yang, Y. M. (2006). Genetic diversity among flue-cured tobacco (Nicotiana tabacum L.) revealed by amplified fragment length polymorphism. Botanical Studies, 47, 223-229. 
Table 1. Name and other details of 19 tobacco cultivars used in this AFLP analysis.

\begin{tabular}{|c|c|c|c|c|c|}
\hline $\begin{array}{l}\text { Sample } \\
\text { no. }\end{array}$ & Cultivar name & $\begin{array}{l}\text { Cultivar } \\
\text { group }\end{array}$ & $\begin{array}{l}\text { Group } \\
\text { type }\end{array}$ & Curing method & $\begin{array}{l}\text { Area of cultivation } \\
\text { (province/region) }\end{array}$ \\
\hline 1 & K326 & Imported & Virginia & Flue-cured & Lamphun / North \\
\hline 2 & PV09 & Imported & Virginia & Flue-cured & Chiang Rai / North \\
\hline 3 & PVH03 & Imported & Virginia & Flue-cured & Phayao / North \\
\hline 4 & Samsun & Imported & Turkish & Sun-cured & Nakhon Phanom / Northeast \\
\hline 5 & Xanthiyaka & Imported & Turkish & Sun-cured & Nakhon Phanom / Northeast \\
\hline 6 & TN86 & Imported & Burley & Air-cured & $\begin{array}{l}\text { Maejo Tobacco Experiment } \\
\text { Station / Chiang Mai / North }\end{array}$ \\
\hline 7 & TN90 & Imported & Burley & Air-cured & $\begin{array}{l}\text { Maejo Tobacco Experiment } \\
\text { Station / Chiang Mai / North }\end{array}$ \\
\hline 8 & TN97 & Imported & Burley & Air-cured & $\begin{array}{l}\text { Maejo Tobacco Experiment } \\
\text { Station / Chiang Mai / North }\end{array}$ \\
\hline 9 & White gold & Local & - & Sun-and-air-cured & Nong Khai / Northeast \\
\hline 10 & $\begin{array}{l}\text { K326 } \\
\text { Phuen-mueang }\end{array}$ & Local & - & Sun-and-air-cured & Nong Khai / Northeast \\
\hline 11 & E-dum & Local & - & Sun-and-air-cured & Phetchabun / Northeast \\
\hline 12 & Kariang & Local & - & Sun-and-air-cured & Kanchanaburi / Central \\
\hline 13 & Kan & Local & - & Sun-and-air-cured & Suphan Buri / Central \\
\hline 14 & Lao-dong & Local & - & Sun-and-air-cured & Suphan Buri / Central \\
\hline 15 & Hangkai & Local & - & Sun-and-air-cured & Phayao / North \\
\hline 16 & $\mathrm{Phu}$ & Local & - & Sun-and-air-cured & Nong Khai / North \\
\hline 17 & Ya-glai & Local & - & Sun-and-air-cured & Nakorn Si Thammarat / South \\
\hline 18 & Bai-lai & Local & - & Sun-and-air-cured & Lop Buri / Central \\
\hline 19 & Bai-tang & Local & - & Sun-and-air-cured & Lop Buri/Central \\
\hline
\end{tabular}

Table 2. The numbers of amplified bands and degrees of polymorphism from the AFLP analyses.

\begin{tabular}{|c|c|c|c|c|}
\hline $\begin{array}{c}\text { Primer } \\
\text { combination }\end{array}$ & $\begin{array}{c}\text { Amplified } \\
\text { bands }\end{array}$ & $\begin{array}{c}\text { Polymorphic } \\
\text { bands }\end{array}$ & $\begin{array}{c}\text { Monomorphic } \\
\text { bands }\end{array}$ & $\begin{array}{c}\text { Polymorphic } \\
\text { percentage (\%) }\end{array}$ \\
\hline $\mathrm{E}_{\mathrm{AAG}} / \mathrm{M}_{\mathrm{CAA}}$ & 35 & 19 & 16 & 54.3 \\
$\mathrm{E}_{\mathrm{AAG}} / \mathrm{M}_{\mathrm{CGC}}$ & 45 & 35 & 10 & 77.8 \\
$\mathrm{E}_{\mathrm{ACT}} / \mathrm{M}_{\mathrm{CAG}}$ & 59 & 49 & 10 & 83.1 \\
\hline Total number & 139 & 103 & 36 & 74.1 \\
Average & 46 & 34 & 12 & 73.9 \\
\hline
\end{tabular}




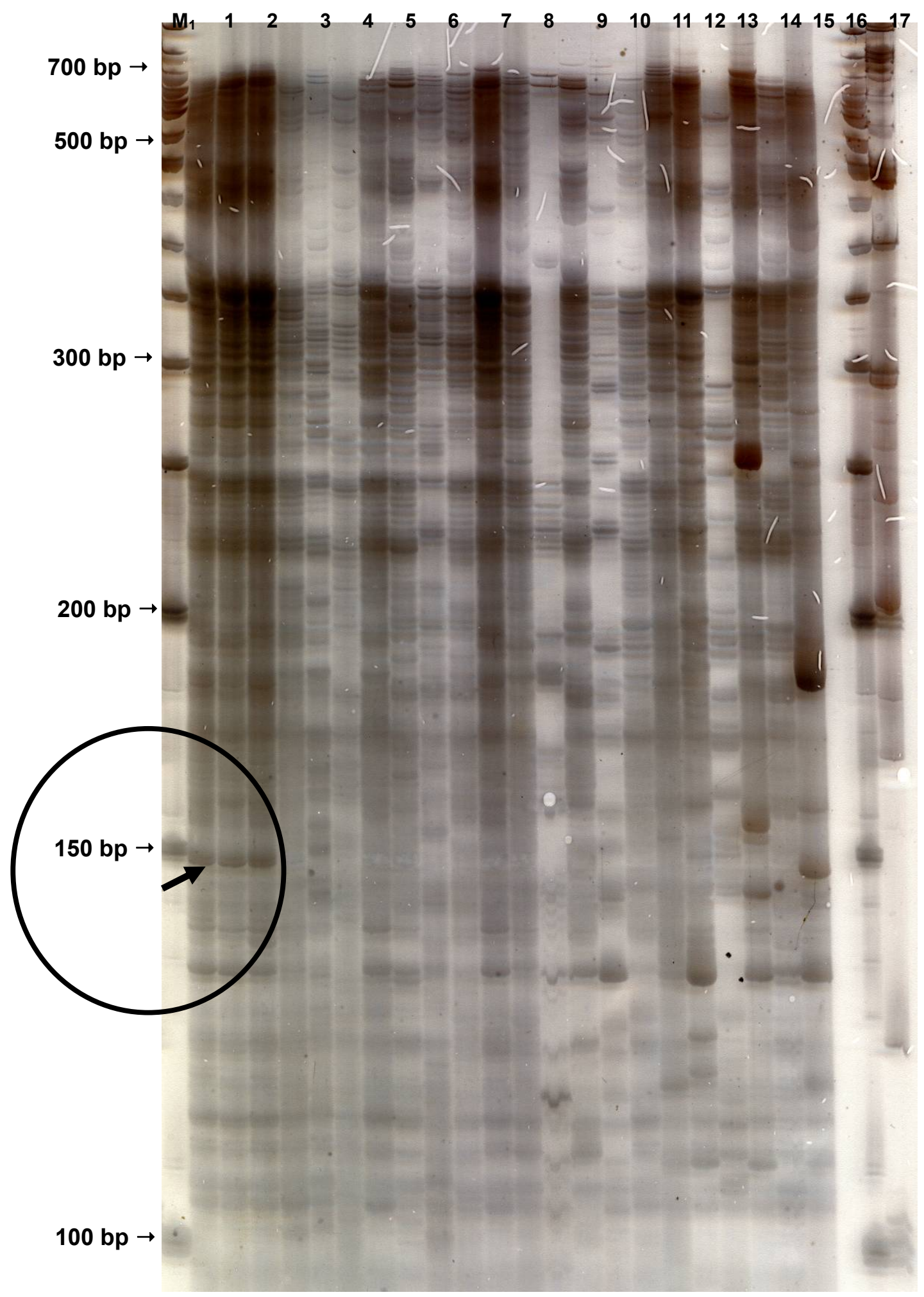

Figure 1. AFLP profile of cured leaf samples of tobacco cultivars using $\mathrm{E}_{\mathrm{ACT}} / \mathrm{M}_{\mathrm{CAG}}$ selective primers. An arrow in the circle indicates a 150-bp specific band AFLP fragment for Virginia type of the imported cultivars. 50 bp and 100 bp DNA ladder markers (lanes $\mathrm{M}_{1}$ and $\mathrm{M}_{2}$, respectively) were used for fragment-size estimation. Lanes numbers 1-22 correspond to tobacco cultivars K326, PV09, PVH03, KY14, TN86, TN90, TN97, Samsun, Xanthiyaka, White gold, K326 Phuen-mueang, E-dum, E-lueang, Phu, Hangkai, Ya-glai, Bai-lai, Kariang, Ya-mueang, Kan, Bai-tang and Lao-dong, respectively. (Note: KY14, E-lueang and Ya-mueang cultivars (lanes 4, 13 and 18, respectively) were not clearly amplified with the other two primer pairs, and therefore were not included in the genetic relationship analysis.) 


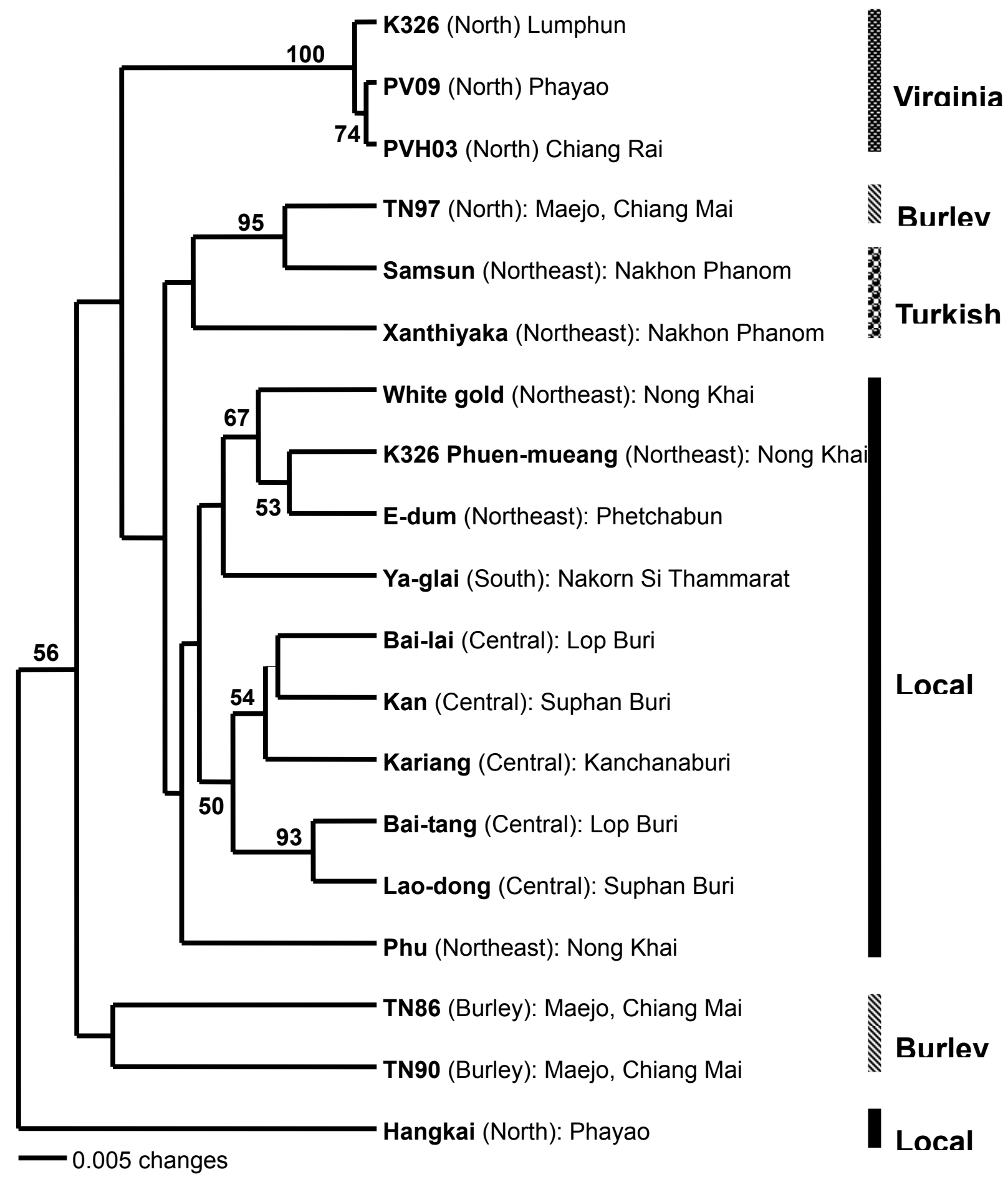

Figure 2. Genetic relationship tree from 139 AFLP bands of 19 tobacco cultivars using UPGMA technique. Numbers along branches are bootstrap-supporting values generated after 1,000 replications. The bootstrap values less than $50 \%$ were not shown on the tree. 